\title{
Are the effects of methylphenidate uncertain?
}

\author{
S. Cortese $\mathrm{e}^{1,2,3,4, *}$ \\ ${ }^{1}$ Academic Unit of Psychology, University of Southampton, Southampton, UK \\ ${ }^{2}$ Solent NHS Trust, Southampton, UK \\ ${ }^{3}$ Clinical and Experimental Sciences (CNS and Psychiatry), Faculty of Medicine, University of Southampton, Southampton, UK \\ ${ }^{4}$ The Child Study Center, New York University, Langone Medical Center, New York
}

\begin{abstract}
Objectives. A recent systematic review and meta-analysis of randomised controlled trials of methylphenidate (MPH) in children and adolescents by a Cochrane group, led by Storebø, raised concern around the level of evidence supporting the use of this medication for attention-deficit/hyperactivity disorder (ADHD) in children and adolescents. This led to several critical responses from a number of ADHD experts.
\end{abstract}

Methods. This paper reviews the conclusions reached from the Storebø meta-analysis by a critical analysis of methodologies used along with drawing on extant literature.

Results. The controversy raised by the Cochrane meta-analysis should lead to a balanced reflection on the research priorities and needs for the field.

Conclusions. It is hoped the controversy will ultimately lead to improve the quality of the research on the efficacy, effectiveness and tolerability of MPH for ADHD.

Received 22 June 2017; Revised 04 January 2018; Accepted 29 March 2018; First published online 6 May 2018

Key words: ADHD, amphetamines, antomoxetine, methylphenidate, stimulants.

\section{Introduction}

Whilst several non-pharmacological strategies have been proposed for attention-deficit/hyperactivity disorder (ADHD) (Sonuga-Barke et al. 2013; Stevenson et al. 2014; Cortese et al. 2015, 2016), pharmacological treatment is an important component of the multimodal treatment recommended for this disorder (Cortese et al. 2017). Medications for ADHD comprise psychostimulant [e.g. methylphenidate (MPH) and amphetamine derivatives] and non-psychostimulant drugs (e.g. atomoxetine, clonidine and guanfacine) (Cortese \& Rosello-Miranda, 2017). MPH is the most commonly used psychostimulant for ADHD in many countries, where it has been used for several decades (Maia et al. 2014).

Despite previous systematic reviews and metaanalyses pointing to high effect sizes, when considering the efficacy of $\mathrm{MPH}$ for the reduction of ADHD symptoms in the short term (e.g. Schachter et al. 2001; Van der Oord et al. 2008; Koesters et al. 2009; Castells et al. 2011), a recent systematic review and metaanalysis by a Cochrane group led by Storebø (Storebo et al. 2015) questioned the evidence base for the efficacy and tolerability of $\mathrm{MPH}$ for ADHD in children and

* Address for correspondence: S. Cortese, M.D., Ph.D., Academic Unit of Psychology, University of Southampton, Southampton, UK.

(Email: samuele.cortese@gmail.com)

This paper is based on a lecture at the International Conference: 'The ADHD Tsunami? Global perspectives' University College of Dublin, 14 October 2016 adolescents. This generated a strong and passionate reaction from the ADHD scientific community (e.g. Banaschewski et al. 2016; Romanos et al. 2016; Hoekstra \& Buitelaar, 2016).

The aim of this paper is to summarise the findings and conclusions of the Cochrane meta-analysis, to present the key critiques to it, and to consider it in the broader context of the evidence base for the efficacy and tolerability of $\mathrm{MPH}$.

\section{The Cochrane meta-analysis}

The aim of the work by the Cochrane group led by Storebø was to systematically review and meta-analyse randomised controlled trials (RCTs) reporting outcomes related to the efficacy and/or tolerability of $\mathrm{MPH}$ in children and/or adolescents with ADHD. Storebø et al. included RCTs of MPH for children and adolescents with ADHD (defined based on DSM-III, III-R, IV, IV-TR, 5 or ICD-9 or 10), with or without psychiatric comorbidities, irrespective of language, publication year, publication type or publication status. Furthermore, it was required that at least $75 \%$ of participants in each trial had an IQ $>70$. The primary outcomes were ADHD symptoms, assessed by teachers. The authors also recorded serious adverse events reported in the studies as a primary outcome, with less severe adverse vents being considered as a secondary outcome measure. Additional secondary outcomes were general behaviour in school and at home, as rated 
by psychometric instruments such as the Child Behaviour Checklist (e.g. CBCL), and quality of life, as measured by psychometric instruments such as the Child Health Questionnaire (e.g. CHQ).

In line with the state of the art recommendations to rate the study risk of bias (RoB) and overall evidence quality, Storebret al. used the Cochrane RoB tool to rate the RoB of individual RCTs included in their systematic review, and the GRADE system to assess the overall quality of the evidence. The standard RoB includes the following six items, which are rated as at low, unclear or high risk for each study: selection bias (random sequence generation; allocation concealment); performance bias (blinding participants/personnel); detection bias (blinding assessor); attrition bias (incomplete outcome data); reporting bias (selective reporting); other bias. Of note, Storebø et al. added a 7th item, that is not formally included in the RoB, that is, vested interest, related to industry funding of the study and authors' conflict of interest, in particular due to relationship with drug companies. The authors considered that a study was at overall high RoB if any one of the seven items received a score of either 'high' or 'unclear risk' of bias.

The GRADE system is based on the assessment of the within-trial RoB: directness of the evidence, heterogeneity of the data, precision of effect estimates and risk of publication bias.

Storebø et al. found 38 parallel-group trials (including a total of 5111 participants) and 147 cross-over trials (comprising a total of 7134 participants) pertinent for their systematic review. The average duration of the included RCTs was 75 days.

The authors found that the effect size for the efficacy of MPH on the primary outcome (ADHD symptoms rated by teachers) was 0.77 (0.64-0.90), which corresponds to a mean difference (MD) of -9.6 points [ $95 \%$ confidence interval (CI) -13.75 to -6.38 ] on the ADHD Rating Scale (ADHD-RS). Of note, Storebø et al. point out that a change of 6.6 points on the ADHD-RS is considered clinically to represent the minimal relevant difference. The effect size for the primary efficacy measure is indeed one of the highest effect sizes found in psychiatry, and more generally across medical disciplines (Leucht et al. 2012).

The authors also found no evidence that $\mathrm{MPH}$ was associated with an increase in serious adverse events [risk ratio (RR) $0.98,95 \%$ CI 0.44 to 2.22]. As for the secondary outcomes, teacher-rated general behaviour (SMD $-0.87,95 \% \mathrm{CI}-1.04$ to -0.71 ) and quality of life (SMD 0.61, 95\% CI 0.42 to 0.80 ) were improved with $\mathrm{MPH}$. Regarding secondary outcomes related to tolerability, the authors found a $29 \%$ increase in the overall risk of any non-serious adverse events [RR: 0.98, 95\% CI 0.44 to 2.22]. The most frequent adverse events were sleep disturbance and appetite decrease. More specifically, children in the MPH group were at $60 \%$ greater risk for trouble sleeping/sleep problems (RR $1.60,95 \%$ CI 1.15 to 2.23 ; 13 trials, 2416 participants), and $266 \%$ greater risk for decreased appetite (RR 3.66, $95 \%$ CI 2.56 to 5.23; 16 trials, 2962 participants) than children in the control group.

Based on their summative analysis, Storebø et al. deemed 'all 185 trials were assessed to be at high risk of bias' and that 'the quality of the evidence was very low for all outcomes'

Therefore, the Cochrane group concluded that the low quality of the underpinning evidence means that we cannot be certain of the magnitude of the effects' and that 'If MPH treatment is considered, clinicians might need to use it for short periods, with careful monitoring of both benefits and harms, and cease its use if no evidence of clear improvement of symptoms is noted, or if harmful effects appear'. Finally, Storebø et al. recommended the use of nocebo in future studies, to reduce the risk of unblinding.

\section{Critiques to the Cochrane meta-analysis}

As mentioned, the Cochrane meta-analysis generated a series of critical reactions from several ADHD experts across the world, both in scientific journals and in blogs, to which Storebø et al. have systematically replied.

The main critiques have focused around:

(1) An idiosyncratic and too stringent approach to rate the RoB of individual studies. In particular, it has been highlighted that the RoB in the meta-analysis by Storebø et al. included the vested interest item, which is not part of the standard Cochrane RoB (Banaschewski et al. 2016). Storebø et al. replied that there is evidence, based on work from Andreas Lundh et al. (cited in Storebo et al. 2015) that 'there are many subtle mechanisms through which sponsorship and conflict of interest may influence intervention effects on outcomes.' However, it has been pointed out (Banaschewski et al. 2016) that there is evidence showing that vested interests do not impact the overall RoB of a study. It is fair to conclude that evidence on this issue is far from being conclusive. In addition, it has been highlighted that considering the overall quality of a study as LOW just because at least one item of the RoB was Unclear may be too stringent. Whilst Storebø et al. cited evidence supporting this, other meta-analyses (e.g. Catala-Lopez et al. 2017), rated the RoB as high if at least one item was rated as high risk; if the risk was rated as 'unclear', this did not result in an overall high study RoB. This is important to consider since often times items in the $\mathrm{RoB}$ are rated Unclear just because of poor reporting, when indeed the risk could be lower if 
full information from the paper were available. Finally, in terms of GRADE, Storebø et al. downgraded the quality of evidence by one point for inconsistency of effects (heterogeneity) and by two points for high RoB. Both these decisions are questionable. As for heterogeneity, $I^{2}$ for the metaanalysis of the main outcome was $37 \%$ for the primary outcome measure. The Cochrane Handbook suggests that heterogeneity up to $40 \%$ may not be important. Clearly, there is a certain level of subjectivity and uncertainty in the use of the threshold, which may lead to discrepant views.

(2) Inclusion of studies, such as the Multimodal Treatment of ADHD, with no placebo/no treatment, or studies in pre-schoolers (for which the effects of MPH are notoriously less evident), which is likely to under-estimate the effect of $\mathrm{MPH}$. Although Storebø et al. pointed out that this was done according to their pre-published protocol, it goes without saying that issues in the protocol are not less concerning than issues in the meta-analysis per se. More importantly, even removing these studies, the assessment of study bias and evidence quality (see previous point) is still problematic.

(3) An emphasis on non-serious adverse events. Indeed, overestimating the adverse events associated with a medication may result in individuals with ADHD being exposed to harm. However, it may lead to the patient not benefitting from effective medications, if the potential adverse events are overestimated, limiting children's access to effective treatment for ADHD, which has serious implications, given the substantial risks of not treating ADHD. Although, as found by Storebø et al., sleep disorders and decrease of appetite are more frequent with MPH compared with placebo, they tend to be transitory in most cases and can be clinically managed (Cortese et al. 2013), but this was not highlighted in the Cochrane review.

(4) Errors in computation of effect sizes. After the European ADHD Guidelines Group highlighted them, Storebø et al. acknowledged these mistakes, stating that they will be corrected in further revisions of the meta-analysis. Overall, these were minor mistakes.

(5) It has been pointed out that the use of a nocebo would be highly unethical in children. Whilst Storebø et al. suggested that it should be used initially for adults, the issue of its use in children is still problematic.

Ultimately, it appears that the controversy around the level of the evidence base for $\mathrm{MPH}$ is, at least in part, linked to the lack of consensus on how to rate important aspects related to possible RoB of studies and more in the cut off to adopt when using the GRADE to appraise the evidence.

\section{Evidence base for ADHD: the broader context}

It should be considered that the duration of the RCTs included in the Cochrane review was overall short (average 75 days), which clearly is not informative for clinicians who see patients usually for many years, given the chronic nature of ADHD in the majority of patients. Overall, readers should consider not only evidence from RCTs, but also from other types of designs and studies. Whilst it is unethical to run RCTs for long periods, it is useful to consider evidence form withdrawal design RCTs (which are still few in the field) and from epidemiological studies. Indeed, large epidemiological studies, published in very high-profile journal, show the long-term benefits of MPH. For instance, a study published in the New England Journal of Medicine (Lichtenstein et al. 2012) in 25656 patients with a diagnosis of ADHD found that, compared with non-medication periods, there was a significant reduction of $32 \%$ in the criminality rate for men (adjusted hazard ratio, $0.68 ; 95 \%$ CI 0.63 to 0.73 ) and $41 \%$ for women (hazard ratio, $0.59 ; 95 \%$ CI 0.50 to 0.70) when they were treated with MPH. Furthermore, large epidemiological studies have found no evidence for an association between stimulants (including $\mathrm{MPH}$ ) and severe cardiovascular effects. A large study (Cooper et al. 2011) of 1200438 children and young adults between the ages of 2 and 24 years found no evidence that current use of a medication for ADHD was associated with an increased risk of severe cardiovascular events (sudden cardiac death, acute myocardial infarction and stroke), although the upper limit of the $95 \% \mathrm{CI}=0.31$ to 1.85 ) indicated that a doubling of the risk could not be ruled out. Another large study (Habel et al. 2011) in 443198 adults and an additional one (Schelleman et al. 2011) in 241, 417 children (3-17 years) concur with the previous one confirming that ADHD drugs use is not associated with increased risk of severe cardiovascular events. Although these large studies are reassuring, a more a recent study found an increased risk of severe cardiovascular events in the first 2 weeks of treatment (Shin et al. 2016), although the methodology of this study has been criticised (BMJ, 2016).

Finally, when it comes to the evidence on the use of $\mathrm{MPH}$, one should also consider evidence on the neurobiological underpinnings for the action of MPH. Of note, a meta-analysis of functional magnetic resonance imaging studies suggested that MPH normalises brain activity in key brain regions (bilateral inferior frontal cortex/insula) affected in the disorder (Rubia et al. 2014).

Overall, readers should consider not only evidence from RCTs, but also from other types of designs and studies. 


\section{Conclusions}

The meta-analysis by Storebø generated a strong controversy. It appears that some of the issues might be attributed to clinicians' lack of consensus as to the methodology used by the Storebø group and the potential for subjective choices on how each study was rated for both quality and potential bias. It is hoped that the very visible and immediate response to this review, will be an opportunity for the field to think of how to design and conduct better, high quality studies and how to improve the methods to appraise the level of evidence.

\section{Financial Support}

No financial support was provided for this work.

\section{Conflicts of Interest}

The author has no conflicts of interest to disclose.

\section{Ethical Standards}

The authors assert that all procedures contributing to this work comply with the ethical standards of the relevant national and institutional committee on human experimentation with the Helsinki Declaration of 1975, as revised in 2008.

\section{References}

Banaschewski T, Buitelaar J, Chui CS, Coghill D, Cortese S, Simonoff E, Wong IC (2016). Methylphenidate for ADHD in children and adolescents: throwing the baby out with the bathwater. Evidence-Based Mental Health 19, 97-99.

BMJ. (2016). Rapid Responses (http:/ / www.bmj.com/ content/353/bmj.i2550/rapid-responses). Accessed 30 December 2017.

Castells X, Ramos-Quiroga JA, Rigau D, Bosch R, Nogueira M, Vidal X, Casas M (2011). Efficacy of methylphenidate for adults with attention-deficit hyperactivity disorder: a metaregression analysis. CNS Drugs 25, 157-169.

Catalá-López F, Hutton B, Núñez-Beltrán A, Page MJ, Ridao M, Macías Saint-Gerons D, Catalá MA, Tabarés-Seisdedos R, Moher D (2017). The pharmacological and nonpharmacological treatment of attention deficit hyperactivity disorder in children and adolescents: a systematic review with network meta-analyses of randomised trials. PLoS One 12, e0180355.

Cooper WO, Habel LA, Sox CM, Chan KA, Arbogast PG, Cheetham TC, Murray KT, Quinn VP, Stein CM, Callahan ST, Fireman BH, Fish FA, Kirshner HS, O’Duffy A, Connell FA, Ray WA (2011). ADHD drugs and serious cardiovascular events in children and young adults. New England Journal of Medicine 365, 1896-1904.

Cortese S, Adamo N, Mohr-Jensen C, Hayes AJ, Bhatti S, Carucci S, Del GC, Atkinson LZ, Banaschewski T, Simonoff E, Zuddas A, Barbui C, Purgato M,
Steinhausen H-C, Shokraneh F, Xia J, Cipriani A, Coghill D; European ADHD Guidelines Group (EAGG) (2017). Comparative efficacy and tolerability of pharmacological interventions for attention-deficit/hyperactivity disorder in children, adolescents and adults: protocol for a systematic review and network meta-analysis. BMJ Open 7, e013967.

Cortese S, Ferrin M, Brandeis D, Buitelaar J, Daley D, Dittmann RW, Holtmann M, Santosh P, Stevenson J, Stringaris A, Zuddas A, Sonuga-Barke EJ; European ADHD Guidelines Group (EAGG) (2015). Cognitive training for attention-deficit/hyperactivity disorder: metaanalysis of clinical and neuropsychological outcomes from randomized controlled trials. Journal of American Academy of Child Adolescent Psychiatry 54, 164-174.

Cortese S, Ferrin M, Brandeis D, Holtmann M, Aggensteiner P, Daley D, Santosh P, Simonoff E, Stevenson J, Stringaris A, Sonuga-Barke EJ; European ADHD Guidelines Group (EAGG) (2016). Neurofeedback for attention-deficit/ hyperactivity disorder: meta-analysis of clinical and neuropsychological outcomes from randomized controlled trials. Journal of American Academy of Child and Adolescent Psychiatry 55, 444-455.

Cortese S, Holtmann M, Banaschewski T, Buitelaar J, Coghill D, Danckaerts M, Dittmann RW, Graham J, Taylor E, Sergeant J; European ADHD Guidelines Group (2013). Practitioner review: current best practice in the management of adverse events during treatment with ADHD medications in children and adolescents. Journal of Child Psychology and Psychiatry 54, 227-246.

Cortese S, Rosello-Miranda R (2017). [Treatments for children and adolescents with attention deficit hyperactivity disorder: what is the evidence base to date?]. Nature Reviews Neurology 64, S3-S7.

Habel LA, Cooper WO, Sox CM, Chan KA, Fireman BH, Arbogast PG, Cheetham TC, Quinn VP, Dublin S, Boudreau DM, Andrade SE, Pawloski PA, Raebel MA, Smith DH, Achacoso N, Uratsu C, Go AS, Sidney S, Nguyen-Huynh MN, Ray WA, Selby JV (2011). ADHD medications and risk of serious cardiovascular events in young and middle-aged adults. JAMA 306, 2673-2683.

Hoekstra PJ, Buitelaar JK (2016). Is the evidence base of methylphenidate for children and adolescents with attention-deficit/hyperactivity disorder flawed? European Child and Adolescent Psychiatry 25, 339-340.

Koesters M, Becker T, Kilian R, Fegert JM, Weinmann S (2009). Limits of meta-analysis: methylphenidate in the treatment of adult attention-deficit hyperactivity disorder. Journal of Psychopharmacology 23, 733-744.

Leucht S, Hierl S, Kissling W, Dold M, Davis JM (2012). Putting the efficacy of psychiatric and general medicine medication into perspective: review of meta-analyses. British Journal of Psychiatry 200, 97-106.

Lichtenstein P, Halldner L, Zetterqvist J, Sjolander A, Serlachius E, Fazel S, Långström N, Larsson H (2012). Medication for attention deficit-hyperactivity disorder and criminality. New England Journal of Medicine 367, 2006-2014.

Maia CR, Cortese S, Caye A, Deakin TK, Polanczyk GV, Polanczyk CA, Rohde LA (2014). Long-term efficacy of 
methylphenidate immediate-release for the treatment of childhood ADHD: a systematic review and meta-analysis. Journal of Attention Disorder 21, 3-13.

Romanos M, Coghill D, Gerlach M, Becker K, Holtmann M, Dopfner M, Banaschewski T (2016). Check and double check - the Cochrane review by Storebo et al. (2015) is indeed flawed. Zeitschrift Fur Kinder Und Jugendpsychiatrie Und Psychotherapie 44, 336-337.

Rubia K, Alegria AA, Cubillo AI, Smith AB, Brammer MJ, Radua J (2014). Effects of stimulants on brain function in attention-deficit/hyperactivity disorder: a systematic review and meta-analysis. Biological Psychiatry 76, 616-628.

Schachter HM, Pham B, King J, Langford S, Moher D (2001). How efficacious and safe is short-acting methylphenidate for the treatment of attention-deficit disorder in children and adolescents? A meta-analysis. CMAJ 165, 1475-1488.

Schelleman H, Bilker WB, Strom BL, Kimmel SE, Newcomb C, Guevara JP, Daniel GW, Cziraky MJ, Hennessy S (2011). Cardiovascular events and death in children exposed and unexposed to ADHD agents. Pediatrics 127, 1102-1110.

Shin JY, Roughead EE, Park BJ, Pratt NL (2016). Cardiovascular safety of methylphenidate among children and young people with attention-deficit/hyperactivity disorder (ADHD): nationwide self controlled case series study. BMJ 353, i2550.

Sonuga-Barke EJ, Brandeis D, Cortese S, Daley D, Ferrin M, Holtmann M, Stevenson J, Danckaerts M, van der Oord S,
Döpfner M, Dittmann RW, Simonoff E, Zuddas A, Banaschewski T, Buitelaar J, Coghill D, Hollis C, Konofal E, Lecendreux M, Wong IC, Sergeant J; European ADHD Guidelines Group (2013). Nonpharmacological interventions for ADHD: systematic review and metaanalyses of randomized controlled trials of dietary and psychological treatments. American Journal of Psychiatry 170, 275-289.

Stevenson J, Buitelaar J, Cortese S, Ferrin M, Konofal E, Lecendreux M, Simonoff E, Wong IC, Sonuga-Barke E (2014). Research Review: The role of diet in the treatment of attention-deficit/hyperactivity disorder - an appraisal of the evidence on efficacy and recommendations on the design of future studies. Journal of Child Psychology and Psychiatry 55, 416-427.

Storebo OJ, Ramstad E, Krogh HB, Nilausen TD, Skoog M, Holmskov M, Rosendal S, Groth C, Magnusson FL, Moreira-Maia CR, Gillies D, Buch Rasmussen K, Gauci D, Zwi M, Kirubakaran R, Forsbøl B, Simonsen E, Gluud C (2015). Methylphenidate for children and adolescents with attention deficit hyperactivity disorder (ADHD). Cochrane Database of Systematic Review 11, CD009885.

Van der Oord S, Prins PJ, Oosterlaan J, Emmelkamp PM (2008). Efficacy of methylphenidate, psychosocial treatments and their combination in school-aged children with ADHD: a meta-analysis. Clinical Psychology Review 28, 783-800. 